\title{
In vivo dosimetry for prostate cancer patients using an electronic portal imaging device (EPID); demonstration of internal organ motion
}

\author{
Marco Kroonwijk $^{\mathrm{a}}$, Kasper L. Pasma ${ }^{\mathrm{a}, *}$, Sandra Quint ${ }^{\mathrm{b}}$, Peter C.M. Koper ${ }^{\mathrm{b}}$, \\ Andries G. Visser ${ }^{\mathrm{a}}$, Ben J.M. Heijmen ${ }^{\mathrm{a}}$ \\ ${ }^{a}$ Department of Clinical Physics, Daniel den Hoed Cancer Center/University Hospital Rotterdam, Groene Hilledijk 301, 3075 EA Rotterdam, The Netherlands \\ ${ }^{\mathrm{b}}$ Department of Radiation Oncology, Daniel den Hoed Cancer Center/University Hospital Rotterdam, Groene Hilledijk 301, \\ 3075 EA Rotterdam, The Netherlands
}

Received 6 January 1998; revised version received 21 September 1998; accepted 28 September 1998

\begin{abstract}
Purpose: To investigate the use of a commercially available video-based EPID for in vivo dosimetry during treatment of prostate cancer patients.

Methods: For 10 prostate cancer patients, the inter-fraction variation within measured portal dose images (PDIs) was assessed and measured PDIs were compared with corresponding predicted PDIs based on the planning CT scan of the patient.

Results: For the lateral fields, the average standard deviation in the measured on-axis portal doses during the course of a treatment was $0.9 \%$; for the anterior fields this standard deviation was $2.2 \%$. The difference between the average on-axis measured portal dose and the predicted portal dose was $0.3 \pm 2.1 \%$ ( $1 \mathrm{SD}$ ) for the lateral fields and $0.7 \pm 3.4 \%$ ( $1 \mathrm{SD}$ ) for the anterior fields. Off-axis differences between measured and predicted portal doses were regularly much larger (up to 15\%) and were caused by frequently occurring gas pockets inside the rectum of the patients during treatment or during acquisition of the planning CT scan. The detected gas pockets did sometimes extend into the gross tumour volume (GTV) area as outlined in the planning CT scans, implying a shift of the anterior rectum wall and prostate in the anterior direction (internal organ motion).

Conclusions: The developed procedures for measurement and prediction of PDIs allow accurate dosimetric quality control of the treatment of prostate cancer patients. Comparing measured PDIs with predicted PDIs can reveal internal organ motion. C) 1998 Elsevier Science Ireland Ltd. All rights reserved.
\end{abstract}

Keywords: In vivo dosimetry; Transmission dosimetry; EPID; Portal imaging; Organ motion; Prostate cancer

\section{Introduction}

EPIDs have become a useful tool for the verification and correction of daily patient set-up during radiotherapy treatment $[5,6]$. For this purpose, the position of bony structures in a portal image, relative to the treatment field, is compared with their position in a corresponding simulator image or digitally reconstructed radiograph (DRR).

Portal images also contain dosimetric information; the pixel signals (grey scale values) in portal images are related to the portion of radiation transmitted through the patient and hence also related to the dose absorbed by the patient. Several years ago investigations began to determine whether the video-based SRI-100 EPID (Philips Medical Systems, Crawley, UK) can be used for dosimetric treatment verification. The linearity and stability of this system

\footnotetext{
* Corresponding author.
}

make it very suitable for dosimetric applications $[8,12,14]$. Since 1994, two of these EPIDs have been in daily use for dosimetric quality control of the scanning photon beams of our MM50 Racetrack Microtron (Scanditronix Medical, Uppsala, Sweden) [8]. Recently, an algorithm has been developed to convert a measured EPID image into an absolute portal dose image (PDI), defined as the (transmitted) dose distribution in the plane of the fluorescent screen of the EPID located at $160 \mathrm{~cm}$ from the focus [12,20,22]. Also, a method has been developed for the prediction of PDIs based on the planning CT scan of the patient [11,21]. Comparing PDIs obtained with the EPID during treatment with predicted PDIs can reveal problems like incorrect (dosimetric) performance of the treatment unit, erroneous design, production or application of compensators and deviations of the patient anatomy during the daily treatment fractions from the anatomy during acquisition of the planning $\mathrm{CT}$ scan [30]. 


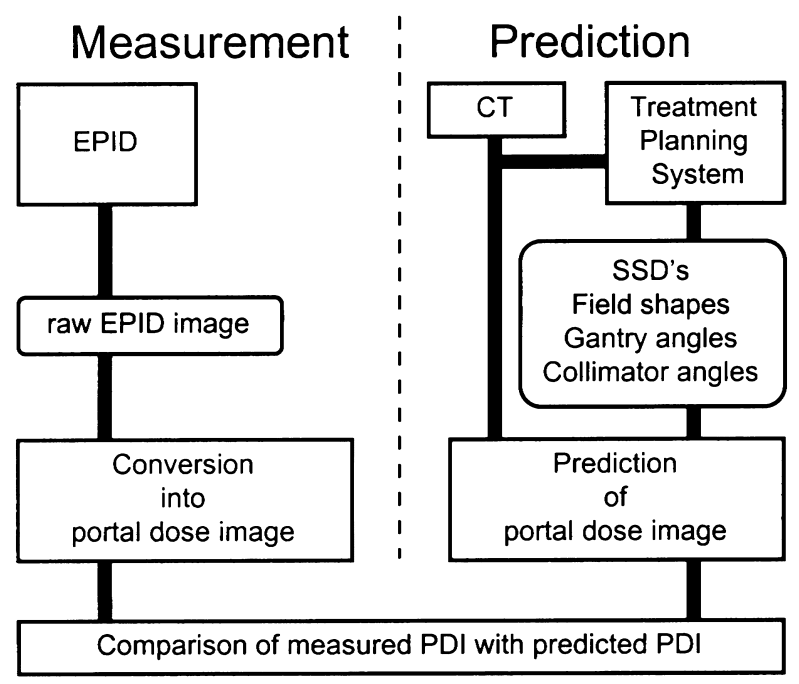

Fig. 1. Schematic overview of the implemented dosimetric treatment verification method (in vivo dosimetry) for comparison of measured and predicted PDIs.

This paper reports on our first clinical experience with in vivo dosimetry using an EPID. For the 10 prostate cancer patients in this study, EPID images were acquired for all treatment fields on every treatment day. The inter-fraction (i.e. day-to-day) variation in measured PDIs was assessed and measured PDIs were compared with a corresponding predicted PDI which was calculated using the planning CT scan of the patient. The obtained data suggest internal organ motion due to observed gas pockets in the rectum of the patients.

\section{Materials and methods}

\subsection{Patients}

Six out of 10 prostate cancer patients in this study parti- cipated in a randomized trial, comparing conventional treatment using rectangular fields with conformal treatment using multi-leaf collimator (MLC) defined fields [15]. One of the patients was treated after a prostatectomy. For all 10 patients a planning CT scan was acquired with a slice distance of $5 \mathrm{~mm}$. The patients were asked to retain a full bladder at the time of acquisition of the planning CT scan and during the daily treatments. No attempts were made to control the rectal contents of the patients. For each patient the visible prostate and the seminal vesicles were outlined manually on all applicable CT slices by the radiation oncologist. The obtained gross tumour volume (GTV) [13] was then extended automatically in three-dimensions (3D) [24,25] with a margin of $15 \mathrm{~mm}$, yielding the planning target volume (PTV) [13]. The treatment plans were designed with the CadPlan 3D treatment planning system (TPS) (Varian-Dosetek, Espoo, Finland). All patients were treated in the supine position with a three-field isocentric technique consisting of one anterior and two lateral oblique fields that were partially delivered with a $60^{\circ}$ wedge inserted. The treatments were performed with the $25 \mathrm{MV}$ photon beam of an MM50 Racetrack Microtron. The dose rate was 300 monitor units (MU) $\mathrm{min}^{-1}$. A total dose of 66 Gy was prescribed to the isocentre and delivered in 33 fractions. Apart from some minor underdosages $(<2 \%)$ in small parts of the superior and inferior ends of the PTV of some patients, the treatment plans fulfilled the ICRU-50 recommendations on dose homogeneity in the PTV [13]. (At present, these underdosages are avoided by using intensity modulated beams [9].)

During treatment, patient set-up was verified and corrected using an off-line correction protocol based on analysis of the position of bony structures in EPID images acquired in the non-wedged portion of each treatment field [5]. Using this protocol the standard deviation of systematic and average random 3D set-up errors are as low as 1.5 and $2.0 \mathrm{~mm}$, respectively. Prior to the analyses of measured

Table 1

On-axis inter-fraction variation in measured PDIs and average on-axis difference between measured and predicted PDIs for lateral and anterior fields separately for 10 prostate cancer patients

\begin{tabular}{|c|c|c|c|c|}
\hline \multirow{2}{*}{$\begin{array}{l}\text { Patient } \\
\text { number }\end{array}$} & \multicolumn{2}{|l|}{ Lateral field } & \multicolumn{2}{|l|}{ Anterior field } \\
\hline & $\begin{array}{l}\text { Difference } \\
\text { between measured } \\
\text { and predicted PDI } \\
(\%)\end{array}$ & $\begin{array}{l}\text { Inter-fraction } \\
\text { variation }(1 \mathrm{SD}) \\
(\%)\end{array}$ & $\begin{array}{l}\text { Difference } \\
\text { between measured } \\
\text { and predicted PDI } \\
(\%)\end{array}$ & $\begin{array}{l}\text { Inter-fraction } \\
\text { variation (1 SD) } \\
(\%)\end{array}$ \\
\hline 1 & -0.1 & 1.2 & -4.2 & 3.5 \\
\hline 2 & 2.7 & 0.5 & 2.0 & 1.6 \\
\hline 3 & 1.2 & 0.3 & -0.3 & 0.2 \\
\hline 4 & 0.5 & 1.1 & 1.5 & 1.7 \\
\hline 5 & 1.9 & 0.6 & 3.6 & 2.6 \\
\hline 6 & -4.6 & 1.0 & 3.7 & 1.3 \\
\hline 7 & 2.2 & 1.1 & 4.2 & 4.2 \\
\hline 8 & 1.6 & 0.2 & -5.6 & 2.8 \\
\hline 9 & -0.8 & 1.5 & 1.3 & 2.2 \\
\hline 10 & -1.3 & 1.1 & 0.8 & 0.7 \\
\hline
\end{tabular}



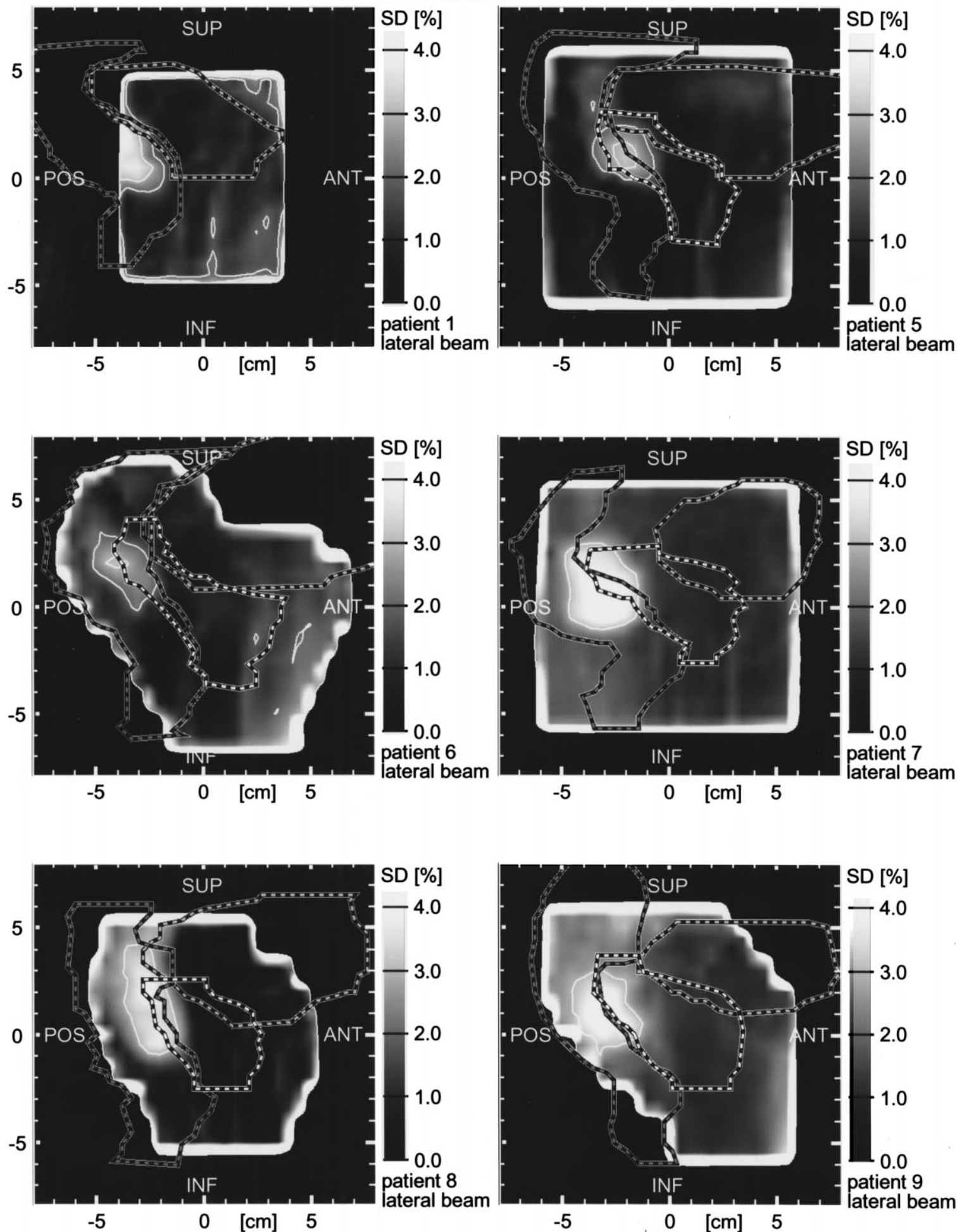

Fig. 2. Observed inter-fraction variations in measured PDIs for the right lateral treatment field of patients 1 and 5-9. Each pixel represents the standard deviation in the observed portal doses during the course of the treatment. The dashed BEV contours represent, from left to right, the rectum, the GTV including prostate and seminal vesicles (not available for patient 1 because of prostatectomy) and the bladder. The presented iso-standard deviation contours connect pixels with standard deviations of $2 \%$ and $3 \%$ for patients 1,5 and 6 . For patients $7-9$ only the $3 \%$ contour is displayed because maximum standard deviations in these images are larger than $4 \%$.

PDIs, the position of the bony structures in all daily measured images was registered with the bony anatomy visible in a corresponding DRR that was derived from the planning CT scan of the patient. 


\subsection{In vivo dosimetry}

For the patients in this study, EPID images were acquired for all treatment fields on every treatment day and the interfraction variation in measured PDIs was assessed. Moreover, measured PDIs were compared with a corresponding predicted PDI calculated using the planning CT scan of the patient; this procedure is schematically shown in Fig. 1. Details on the prediction and measurement of PDIs are discussed in Section 2.2.1 and Section 2.2.2.

\subsubsection{PDI prediction}

The fixed focus-to-fluorescent screen distance of the applied EPID is $160 \mathrm{~cm}$. Due to the resulting large air gap between the patient and the EPID detector $(35-55 \mathrm{~cm})$ the CadPlan TPS cannot be used for accurate prediction of PDIs for this EPID. Therefore, we have developed our own algorithms to predict PDIs using planning CT data of the patient as input [11,16,21]. Primary photon contributions and contributions from photons scattered from the patient onto the EPID are calculated separately and are added to obtain the PDI.

For calculation of the primary component, beam-hardening effects, e.g. due to a flattening filter, a wedge or a compensator, are taken into account. Scatter contribution calculations are basically convolutions of the two-dimensional beam fluence profile entering the patient with scatter kernels, describing the scatter from the patient onto the EPID. Basic input data for the calculations are obtained from a set of transmission measurements through flat polystyrene absorbers for relevant absorber thicknesses, field sizes and distances to the EPID detector [21]. For a wide range of inhomogeneous anthropomorphic phantoms and treatment energies, predicted portal dose values and doses measured with an ionization chamber agree within $1 \%$ (1 SD) $[11,16,21]$.

\subsubsection{PDI measurement}

Portal images were acquired with a commercially available video-based SRI-100 EPID. This EPID basically consists of a fluorescent screen, two mirrors and a charge coupled device $(\mathrm{CCD})$ camera $[1,28]$. In order to reduce detection of high energy electrons generated inside the patient, an extra $1 \mathrm{~mm}$ thick stainless steel plate was mounted on top of the standard fluorescent screen, which consists of a $1.65 \mathrm{~mm}$ thick stainless steel plate coated with a fluorescent layer. The total water equivalent depth of the EPID detector is only slightly smaller than the depth of maximum dose of the applied $25 \mathrm{MV}$ photon beam [22].
The extra build-up layer resulted in a minor decrease in resolution but at the same time led to an increase of the signal-to-noise ratio in portal images. Modifications to the software of the SRI-100 system were performed at the level of the macro command language that comes with the system: (i) the standard image acquisition time was adjusted to fully enclose the irradiation time in order to measure the total delivered dose for each treatment field ( $\leq 58 \mathrm{MU}, 12.8$ s; 59-69 MU, 15.36 s; 70-86 MU, 19.2 s; 87-115 MU, 25.6 s; 116-173 MU, 38.4 s; 174-384 MU, 76.8 s) and (ii) for dosimetric purposes, an additional raw 16-bit image was saved to avoid digitization noise in low grey scale value images $[16,20,22]$. Image enhancement procedures used to obtain adequate image quality for patient set-up verification continued automatically after the raw image was saved. With these modifications acquisition of portal images suitable for in vivo dosimetry could be fully integrated into existing imaging routines for patient set-up verification without introducing an increase in the overall treatment time.

Visible photons produced by the X-ray beam in a point of the fluorescent screen not only generate a signal in the corresponding pixel of the EPID image, but also generate a (much lower) signal in all other pixels. Heijmen et al. [12] have shown that this is due to light scatter from components within the EPID structure onto the CCD chip. PDIs are derived from measured EPID images by removing the contributions from scattered light to the pixel signals in an EPID image using a deconvolution algorithm, as described by Pasma et al. [20,22]. Using this method, derived PDIs generally agree within 1\% (1 SD) with ionization chamber measurements in open, wedged and intensity modulated beams for various anatomical phantoms.

\subsection{EPID stability}

During the study, the stability of the EPID response was verified on a 2-weekly basis by comparing the pixel signals in acquired EPID images with measurements performed with an ionization chamber array positioned at $100 \mathrm{~cm}$ from the focus. Details on the applied set-up and equipment are given in Ref. [8]. During the 4 month period of this study, the variation in absolute on-axis EPID response was $0.7 \%$ (1 SD). The variation in the EPID response relative to the on-axis measured grey scale value was less than $0.2 \%$ ( $1 \mathrm{SD}$ ). Both the observed variations in the absolute on-axis response and in the relative off-axis response are in agreement with the observations of Heijmen et al. [12] and Dirkx et al. [8]. In the analyses of the inter-fraction variation

Fig. 3. The blue, red and yellow contours represent the BEV contours of the rectum, the gross tumour volume and the bladder, respectively; point $(0,0)$ corresponds to the isocentre. (a) Observed inter-fraction variations (1 SD) in the measured portal doses for the right lateral field of patient 8. (b) As in (a) but now for the anterior field. (c) Differences between the portal doses measured during fraction 13 of patient 8 for the right lateral field and the corresponding predicted portal doses. (d) As in (c) but now for the anterior field. (e) Differences between the average measured PDI (average of 27 fractions) and the predicted PDI for the right lateral field of patient 8. (f) As in (e) but now for the anterior field. The applied scale in (c) is different from the scale in (d-f) due to the large deviations in portal dose as measured in fraction 13 in the lateral beam of patient 8 . 


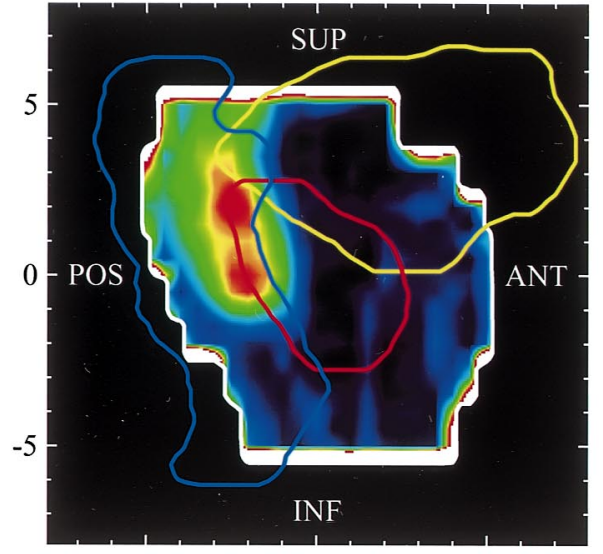

$-5$

[cm] 5

(a)

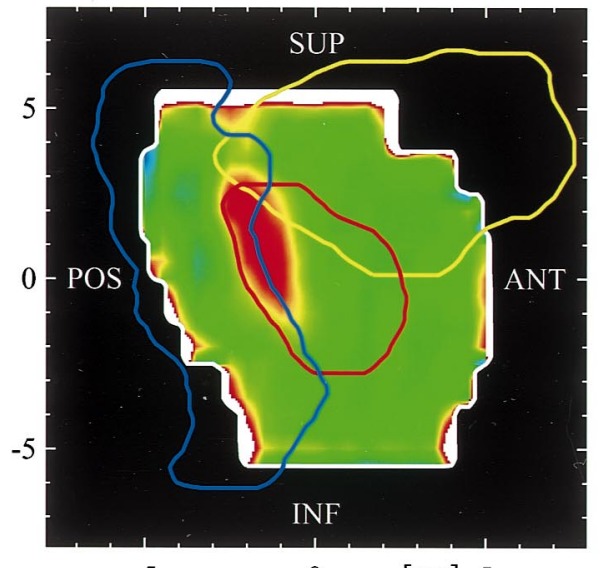

(c)

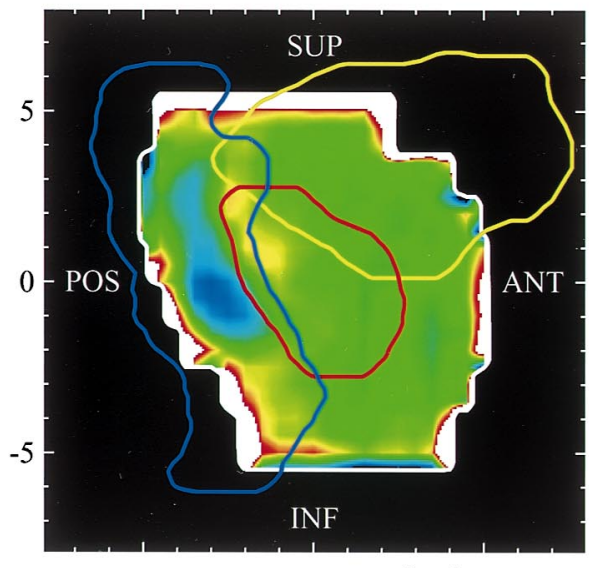

$-5$

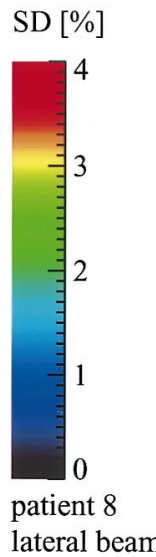

Dm-Dc [\%]

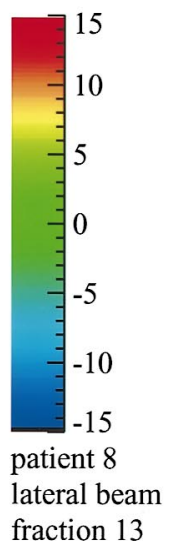

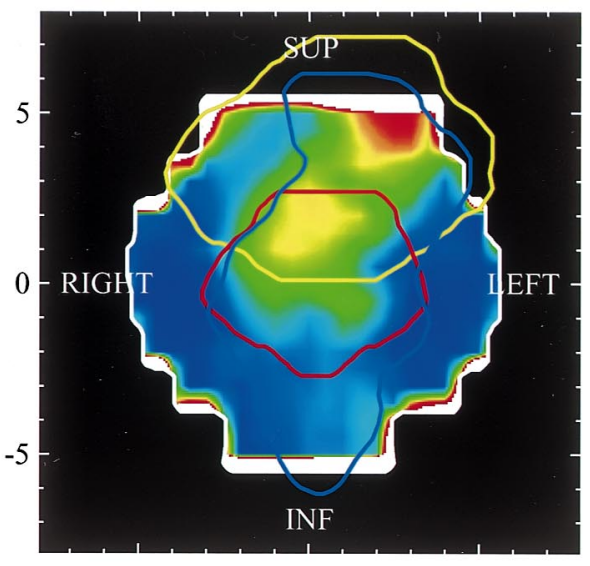

$-5$

[cm] 5 (b)

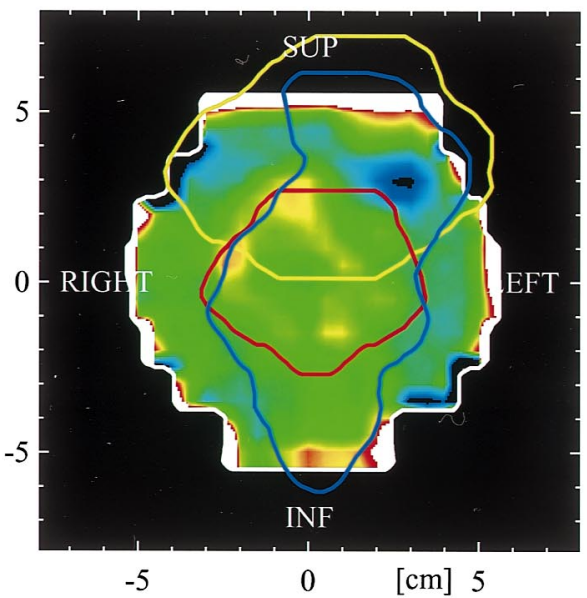

(d)

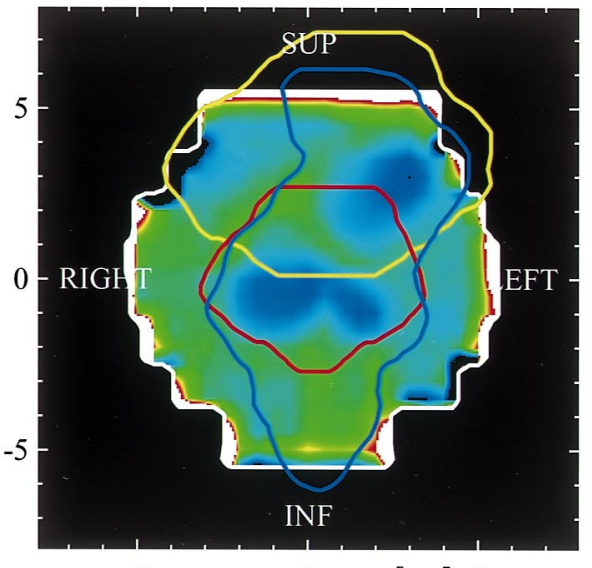

[cm] 5

Dm-Dc [\%]

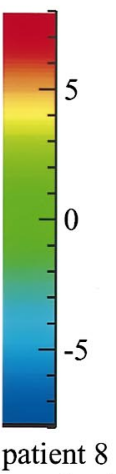

anterior beam fraction 13

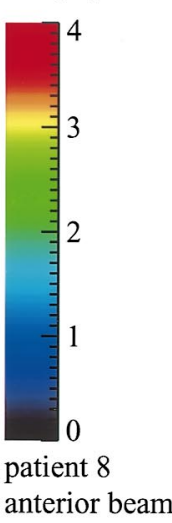

anterior beam

Dm-Dc [\%]

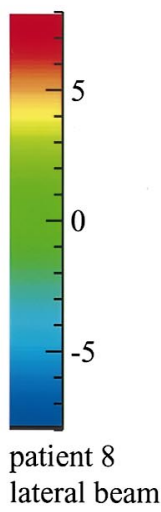

(e)

(f)

Dm-Dc [\%]

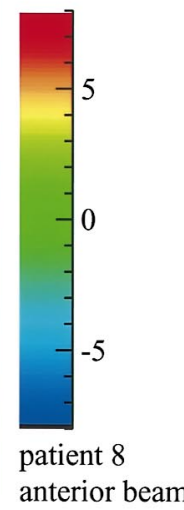

anterior beam 


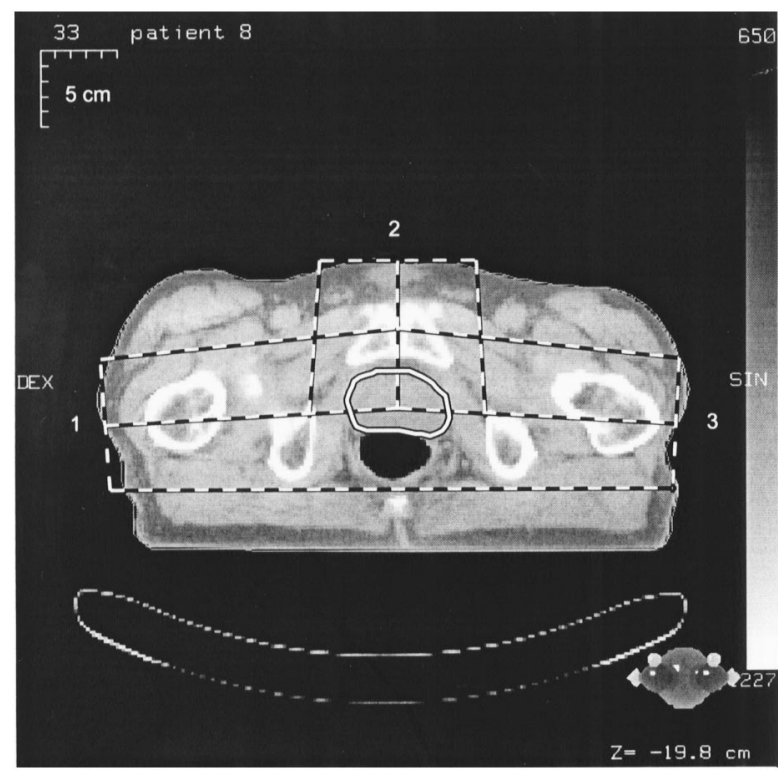

Fig. 4. The planning CT scan of patient 8 used for PDI prediction. The transversal slice shown contains the isocentre. The closed white contour indicates the GTV volume and the dashed lines show the applied beams. Notice the gas pocket inside the rectum.

in the portal doses measured during patient treatment, the reported standard deviations were obtained by subtracting $0.7^{2}$ from the square of each measured standard deviation; this corrected standard deviation is more directly correlated with patient thickness variations.

\section{Results and discussion}

For each patient, acquisition of EPID images was planned for all of the 33 treatment fractions. However, some portal image acquisitions failed, e.g. because of accelerator interlocks or starting image acquisition too late, leaving about 30 successful acquisitions per treatment field per patient.

\subsection{On-axis portal doses}

For each patient and for the lateral and anterior fields separately, the on-axis inter-fraction variation in measured PDIs and the average on-axis differences found between the measured PDIs and the predicted PDI are presented in Table 1. For the lateral fields, the average standard deviation in the on-axis measured portal doses was $0.9 \%$. For the anterior fields the standard deviation was $2.2 \%$. For the 10 patients, the average difference between the on-axis measured portal dose and the predicted portal dose was $0.3 \pm 2.1 \%$ (1 SD) for the lateral fields and $0.7 \pm 3.4 \%$ (1 SD) for the anterior fields.

From the measured set of transmissions through flat water-equivalent absorbers, as used for the PDI prediction algorithm, relationships between radiological thicknesses and portal doses were derived for typical lateral and anterior treatment fields. A variation in radiological thickness of 1 $\mathrm{cm}$, e.g. due to a variation in rectum and/or bladder filling, results in a variation in the transmitted dose of approximately $1.9 \%$ for a lateral field and $3.1 \%$ for an anterior field. This difference between lateral and anterior fields is an explanation for the observed larger standard deviations for the anterior fields. Variations in the on-axis portal dose could also be slightly higher for the anterior fields because for these fields the central ray-line passes both the rectum and the bladder, whereas the central ray-line of the lateral fields does not pass organs with a variable filling.

\subsection{Off-axis portal doses}

For the six patients with the largest inter-fraction variation in measured PDIs, the observed variations for the right lateral field are presented in Fig. 2, together with beam'seye-view (BEV) contours of the GTV (prostate + seminal vesicles), the rectum and the bladder, as derived from the planning CT scan. For patient 8, the inter-fraction variation is also presented in colour in Fig. 3a. For the lateral fields, areas with large variations $(\mathrm{SD}>3 \%$ ) are mainly found for pixels corresponding to rays passing through the planned position of the rectum, the superior/posterior portion of the prostate and the posterior part of the seminal vesicles. For rays passing only through the bladder, the observed variations in measured portal doses are generally much smaller. For the anterior fields, the areas with large variations are also strongly correlating with the BEV contour of the rectum, as depicted in Fig. $3 \mathrm{~b}$ for patient 8 .

For those treatment fractions that show large differences between measured and predicted PDIs, the deviations do mostly occur in the areas of the PDI for which also large inter-fraction variations are observed. For patient 8, differences between the PDI measured during fraction 13 and the corresponding predicted PDI are presented in Fig. 3c,d for the right lateral field and the anterior field, respectively. The maximum difference between the measured and predicted portal dose is $15 \%$, indicating a difference in lateral radiological pathlength with the planning CT scan of about 6 $\mathrm{cm}$. Also for patient 8, Fig. 3e,f shows the difference between the average measured PDI (average of 27 fractions) and the predicted PDI for the right lateral field and the anterior field, respectively.

\subsection{Gas pockets and organ motion}

Observed variations in both lateral and anterior measured PDIs were often due to localized areas of increased portal dose inside the rectum contour and the posterior part of the GTV contour, indicating the presence of gas pockets inside the rectum of the patients of variable size and shape. In the presence of both faeces and a gas pocket, the gas is generally floating on top of the faeces. In Fig. 3c,d, the red spots inside the GTV contour are due to the presence of a large gas pocket inside the rectum of patient 8 during treatment. During acquisition of the planning CT scan, the rectum of 
patient 8 also contained a (smaller) gas pocket (see Fig. 4). Due to this gas pocket, the differences between measured and predicted portal doses inside the BEV contour of the rectum in the lateral view (Fig. 3c) and on-axis differences in the anterior view (Fig. 3d) are relatively small.

The presence of a large gas pocket within the BEV contour of the GTV, as observed in the lateral field (Fig. $3 c$ ), indicates internal organ motion. During fraction 13, the positions of the prostate and seminal vesicles were in disagreement with the planning CT scan. The gas pocket in the rectum of the patient pushed both the anterior part of the rectal wall and the GTV in an anterior direction, yielding a risk for an unintended irradiation of a large part of the rectum and for underdosing the anterior part of the GTV. Similar observations were made for other fractions of patient 8 and also for other patients.

For patient 8, Fig. 3e,f shows for each pixel the difference between the average measured portal dose (average of 27 fractions) and the predicted portal dose for the right lateral field and the anterior field, respectively. The blue spots are due to the presence of the gas pocket during acquisition of the planning CT scan (Fig. 4). The deviations inside the upper part of the GTV contour as presented in Fig. 3e indicate the presence of gas pockets during treatment that resulted in movement of the GTV in an anterior direction.

In recent years, several groups have studied internal organ motion in prostate cancer patients [2$4,7,17,19,23,26,27,29]$. The observed prevalence in this study of gas pockets occurring in the upper part of the rectum close to the superior portion of the prostate and the seminal vesicles (Figs. 2 and 3 ) is in agreement with the presence of internal organ motion in these areas reported in the literature, see e.g. Beard et al. [4] and van Herk et al. [27].

For back-projection methods aiming at calculation of the 3D patient dose distribution from measured portal images (see e.g. Hansen et al. [10] and McNutt et al. [18]), an accurate 3D representation of the patient is required. In this study, we have observed that large deviations between measured and predicted portal doses were always due to differences in patient anatomy during acquisition of the planning CT scan and during the treatment fractions, due to gas pockets in the rectum of the patients. In case of large deviations between predicted and measured PDIs, calculation of the patient dose distribution based on the planning CT scan would therefore have resulted in non-meaningful dose distributions in the corresponding volumes in the patients. In on-going studies we are investigating the applicability of gas pockets visible in EPID images for on-line geometrical verification and correction of the position of the prostate of the patient in the treatment beams. The data presented in this paper do not allow the decision to be made as to whether EPID images can be used for detection of internal organ motion due to variation in the amount of faeces present in the rectum.

\section{Conclusions}

The small average on-axis differences between measured and predicted PDIs and the variation therein indicate that the developed methods and procedures allow accurate dosimetric quality control of the treatment of prostate cancer patients in clinical practice. Large off-axis differences between measured and predicted PDIs have been found to correspond with frequently occurring gas pockets inside the rectum of the patient during treatment or during acquisition of the planning CT scan. In the case where a detected gas pocket extends into the GTV area, as outlined in the planning CT scan, organ motion is evident.

\section{Acknowledgements}

The authors would like to thank Joep Stroom, Maarten Dirkx and Hans de Boer for fruitful discussions and are grateful to the technologists working at the MM50 Racetrack Microtron for the daily acquisition of the images during patient treatment and for the effort that has been made to implement the procedures in clinical practice. This work was financially supported by grant DDHK 94848 awarded by the Dutch Cancer Society.

\section{References}

[1] Althof VGM, de Boer JCJ, Huizenga H, Stroom JC, Visser AG, Swanenburg BN. Physical characteristics of a commercial electronic portal imaging device. Med. Phys. 1996;23:1845-1855.

[2] Balter JM, Sandler HM, Lam K, Bree RL, Lichter AS, Ten Haken RK. Measurement of prostate movement over the course of routine radiotherapy using implanted markers. Int. J. Radiat. Oncol. Biol. Phys. 1995;31:113-118.

[3] Balter JM, Lam KL, Sandler HM, Littles JF, Bree RL, Ten Haken RK. Automated localization of the prostate at the time of treatment using implanted radio-opaque markers: technical feasibility. Int. J. Radiat. Oncol. Biol. Phys. 1995;33:1281-1286.

[4] Beard CJ, Kijewski P, Bussière M, et al. Analysis of prostate and seminal vesicle motion: implications for treatment planning. Int. J. Radiat. Oncol. Biol. Phys. 1996;34:451-458.

[5] Bel A, Vos PH, Rodrigus PTR, et al. High-precision prostate cancer irradiation by clinical application of an off-line patient set-up verification procedure, using portal imaging. Int. J. Radiat. Oncol. Biol. Phys. 1996;35:321-332.

[6] Creutzberg CL, Althof VGM, de Hoog M, et al. A quality control study of the accuracy of patient positioning in irradiation of pelvic fields. Int. J. Radiat. Oncol. Biol. Phys. 1996;34:697-708.

[7] Crook JM, Raymond Y, Salhani D, Yang H, Esche B. Prostate motion during standard radiotherapy as assessed by fiducial markers. Radiother. Oncol. 1995;37:35-42.

[8] Dirkx MLP, Kroonwijk M, de Boer JCJ, Heijmen BJM. Daily dosimetric quality control of the MM50 Racetrack Microtron using an electronic portal imaging device. Radiother. Oncol. 1995;37:55-60.

[9] Dirkx MLP, Heijmen BJM, Korevaar GA, et al. Field margin reduction using intensity modulated X-ray beams formed with a multileaf collimator. Int. J. Radiat. Oncol. Biol. Phys. 1997;38:1123-1129.

[10] Hansen VN, Evans PM, Swindell W. The application of transit dosimetry to precision radiotherapy. Med. Phys. 1996;23:713-721.

[11] Heijmen BJM, Storchi PRM, v.d. Kamer JB. A method for prediction 
of portal dose images (extended abstract). Proceedings of the 11th ICCR. Manchester: North Western Medical Physics Department, 1994:112-113.

[12] Heijmen BJM, Pasma KL, Kroonwijk M, et al. Portal dose measurement in radiotherapy using an electronic portal imaging device (EPID). Phys. Med. Biol. 1995;40:1943-1955.

[13] ICRU Report 50. Prescribing, recording and reporting photon beam therapy. Bethesda, MD: International Commission on Radiotherapy Units and Measurements, 1993.

[14] Kirby MC, Williams PC. The use of an electronic portal imaging device for exit dosimetry and quality control measurements. Int. J. Radiat. Oncol. Biol. Phys. 1995;31:593-603.

[15] Koper PCM, v. Putten WLJ, Stroom JC, et al. Acute morbidity reduction using 3DCRT for prostate carcinoma: a randomised phase III study (abstract). Int. J. Radiat. Oncol. Biol. Phys. 1997;39(Suppl.):191.

[16] Kroonwijk M, Pasma KL, Quint S, Visser AG, Heijmen BJM. Development and clinical implementation of in-vivo dosimetry using an electronic portal imaging device (extended abstract). Proceedings of the 12th ICCR. Salt Lake City, UT: Medical Physics Publishing, 1997:276-278.

[17] Lebesque JV, Bruce AM, Kroes APG, Touw A, Shouman T, van Herk M. Variation in volumes, dose-volume histograms and estimated normal tissue complication probabilities of rectum and bladder during conformal radiotherapy of T3 prostate cancer. Int. J. Radiat. Oncol. Biol. Phys. 1995;33:1109-1119.

[18] McNutt TR, Mackie TR, Reckwerdt P, Paliwal BR. Modelling dose distributions from portal dose images using the convolution/superposition method. Med. Phys. 1996;23:1381-1392.

[19] Melian E, Mageras GS, Fuks Z, et al. Variation in prostate position quantification and implications for three-dimensional conformal treatment planning. Int. J. Radiat. Oncol. Biol. Phys. 1997;38:73-81.

[20] Pasma KL, Kroonwijk M, Visser AG, Heijmen BJM. Portal dose measurements with a video-based electronic portal imaging device using a deconvolution algorithm (extended abstract). Proceedings of the 12th ICCR. Salt Lake City, UT: Medical Physics Publishing, 1997:282-284.
[21] Pasma KL, Heijmen BJM, Kroonwijk M, Visser AG. Portal dose image (PDI) prediction for dosimetric treatment verification in radiotherapy I: an algorithm for open beams. Med. Phys. 1998;25:830-840.

[22] Pasma KL, Kroonwijk M, de Boer JCJ, Visser AG, Heijmen BJM. Accurate portal dose measurement with a fluoroscopic portal imaging device (EPID) for open and wedged beams and for dynamic multileaf collimation. Phys. Med. Biol. 1998;43:2047-2060.

[23] Roeske JC, Forman JD, Mesina CF, et al. Evaluation of changes in the size and location of the prostate, seminal vesicles, bladder and rectum during a course of external beam radiation therapy. Int. J. Radiat. Oncol. Biol. Phys. 1995;33:1321-1329.

[24] Stroom JC, Storchi PRM. Automatic calculation of three-dimensional margins around treatment volumes in radiotherapy planning. Phys. Med. Biol. 1997;42:745-755.

[25] Stroom JC, Korevaar GA, Koper PCM, Visser AG, Heijmen BJM. Multiple 2-dimensional versus 3-dimensional PTV definition in treatment planning for conformal radiotherapy. Radiat. Oncol. 1998;47:297-302.

[26] Ten Haken RK, Forman JD, Heimburger DK, et al. Treatment planning issues related to prostate movement in response to differential filling of the rectum and bladder. Int. J. Radiat. Oncol. Biol. Phys. 1991;20:1317-1324.

[27] van Herk M, Bruce A, Kroes APG, Shouman T, Touw A, Lebesque JV. Quantification of organ motion during conformal radiotherapy of the prostate by three dimensional image registration. Int. J. Radiat. Oncol. Biol. Phys. 1995;33:1311-1320.

[28] Visser AG, Huizenga H, Althof VGM, Swanenburg BN. Performance of a prototype fluoroscopic radiotherapy imaging system. Int. J. Radiat. Oncol. Biol. Phys. 1990;18:43-50.

[29] Vigneault E, Pouliot J, Laverdière J, Roy J, Dorion M. Electronic portal imaging device detection of radio-opaque markers for the evaluation of prostate position during megavoltage irradiation: a clinical study. Int. J. Radiat. Oncol. Biol. Phys. 1997;37:205-212.

[30] Wong JW, Slessinger ED, Hermes RE, Offutt CJ, Roy T, Vannier MW. Portal dose images I: quantitative treatment plan verification. Int. J. Radiat. Oncol. Biol. Phys. 1990;18:1455-1463. 